\title{
POLE PLACEMENT BY STATIC OUTPUT FEEDBACK FOR GENERIC LINEAR SYSTEMS
}

\author{
A. EREMENKO* AND A. GABRIELOV ${ }^{\dagger}$
}

\begin{abstract}
We consider linear systems with $m$ inputs, $p$ outputs and McMillan degree $n$, such that $n=m p$. If both $m$ and $p$ are even, we show that there is a non-empty open (in the usual topology) subset $U$ of such systems, where the real pole placement map is not surjective. It follows that for each system in $U$, there exists an open set of pole configurations, symmetric with respect to the real line, which cannot be assigned by any real static output feedback.
\end{abstract}

Key words. linear systems, static output control feedback, pole placement

AMS subject classifications. 14N10, 14P99, 14M15, 30C99, 26C15

1. Introduction. We consider linear systems $S=(A, B, C)$ described by the equations

$$
\begin{aligned}
& \dot{x}=A x+B u \\
& y=C x
\end{aligned}
$$

Here the state $x$, the input $u$ and the output $y$ are functions of a real variable $t$ (time), with values in $\mathbf{R}^{n}, \mathbf{R}^{m}$ and $\mathbf{R}^{p}$, respectively, the dot denotes the derivative with respect to $t$, and $A, B, C$ are real matrices of sizes $n \times n, n \times m$ and $p \times n$, respectively.

Assuming zero initial conditions and applying the Laplace transform, we obtain

$$
Y(s)=C(s I-A)^{-1} B U(s),
$$

so the behavior of our linear system is described by the rational matrix-function $G(s)=C(s I-A)^{-1} B$ of size $p \times m$ of a complex variable $s$, which is called the (open loop) transfer function of $S$. It is clear that $G(\infty)=0$. The poles of the transfer function are the eigenvalues of the matrix $A$.

For a given $p \times m$ matrix function $G$ with the property $G(\infty)=0$ there exist infinitely many representations of $G$ in the form $G(s)=C(s I-A)^{-1} B$. The smallest integer $n$ over all such representations is called the McMillan degree of $G$.

We consider the possibility to control a given system $S$ by attaching a feedback. This means that the output is sent to the input after a preliminary linear transformation, called a compensator. The compensator may be another system of the form (1.1) (dynamic output feedback) or just a constant matrix (static output feedback). In this paper we consider only static output feedback, referring for the recent results on dynamic output feedback to $[14,11]$.

A static output feedback is described by the equation

$$
u=K y,
$$

where $K$ is an $m \times p$ matrix which is usually called a gain matrix. Eliminating $u$ and $y$ gives

$$
\dot{x}=(A+B K C) x,
$$

${ }^{*}$ Dept. of Math., Purdue Univ., West Lafayette IN, 47907, eremenko@math.purdue.edu, supported by NSF grant DMS-0100512 and Humboldt Foundation

${ }^{\dagger}$ Dept. of Math., Purdue Univ., West Lafayette IN 47907, agabriel@math.purdue.edu, supported by NSF grant DMS-0070666 and James S. McDonnell Foundation. 
whose characteristic polynomial is

$$
\varphi_{K}(s)=\operatorname{det}(s I-A-B K C) .
$$

It is called the closed loop characteristic polynomial.

The pole placement problem is formulated as follows:

Given a system $S=(A, B, C)$, and a set of points $\left\{s_{1}, \ldots, s_{n}\right\}$ in $\mathbf{C}$ (listed with multiplicities) symmetric with respect to the real axis, find a real matrix $K$ such that the zeros of $\varphi_{K}$ are exactly $s_{1}, \ldots, s_{n}$.

For a fixed system $S$, we define the (real) pole placement map

$$
\chi_{S}: \operatorname{Mat}_{\mathbf{R}}(m \times p) \rightarrow \operatorname{Poly}_{\mathbf{R}}(n), \quad \chi_{S}(K)=\varphi_{K},
$$

where $\operatorname{Mat}_{\mathbf{R}}(m \times p)$ is the set of all real matrices of size $m \times p, \operatorname{Poly}_{\mathbf{R}}(n)$ the set of all real monic polynomials of degree $n$, and the polynomial $\varphi_{K}$ is defined in (1.3). Thus to say that for a system $S$, an arbitrary symmetric set of poles can be assigned by a real gain matrix, is the same as to say that the real pole placement map $\chi_{S}$ is surjective. Extending the domain to complex matrices $K$ and the range to complex monic polynomials gives the complex pole placement map

$$
\operatorname{Mat}_{\mathbf{C}}(m \times p) \rightarrow \operatorname{Poly}_{\mathbf{C}}(n),
$$

defined by the same formula as the real one.

It is easy to see that for every $m, n, p$ there are systems for which the pole placement map is not surjective. For example, one can take $B=0$ or $C=0$. A necessary condition of surjectivity proved in [13] is that $S$ is observable and controllable. This is equivalent to saying that the McMillan degree of the transfer function is equal to $n$, the dimension of the state space. Notice that this property is generic: it holds for an open dense subset of the set

$$
\mathfrak{A}=\operatorname{Mat}_{\mathbf{R}}(n \times n) \times \operatorname{Mat}_{\mathbf{R}}(n \times m) \times \operatorname{Mat}_{\mathbf{R}}(p \times n)
$$

of all triples $(A, B, C)$. All topological terms in this paper refer to the usual topology.

In this paper we consider the following problem: for a given triple of integers $(m, n, p)$, does there exist an open dense subset $V \subset \mathfrak{A}$, such that the real pole placement map $\chi_{S}$ is surjective for $S \in V$ ? If this is the case, we say that the real pole placement map is generically surjective for these $m, n$ and $p$.

We briefly recall the history of the problem, referring to a comprehensive survey [2]. The pole placement map defined by (1.3) and (1.4) is a regular map of affine algebraic varieties. Comparing the dimensions of its domain and range, we conclude that $n \leq m p$ is a necessary condition for generic surjectivity of the pole placement map, real or complex. In the complex case, this condition turns is to be also sufficient [7]. To show this, one extends the pole placement map to a regular map between compact algebraic manifolds and verifies that its Jacobi matrix is of full rank. In the case when $n=m p$ we have the following precise result:

Theorem A [1] For $n=m p$, the complex pole placement map is generically surjective. Moreover, it extends to a finite regular map between projective varieties and has degree

$$
d(m, p)=\frac{1 ! 2 ! \ldots(p-1) !(m p) !}{m !(m+1) ! \ldots(m+p-1) !} .
$$


It follows that for a generic system $(A, B, C)$ with $n=m p$ and a generic monic complex polynomial $\varphi$ of degree $m p$, there are $d(m, p)$ complex matrices $K$ such that $\varphi_{K}=\varphi$.

The numbers $d(m, p)$ occur as the solution of the following problem of enumerative geometry: how many $m$-subspaces intersect $m p$ given $p$-subspaces in $\mathbf{C}^{m+p}$ in general position? The answer $d(m, p)$ was obtained by Schubert in 1886 (see, for example, $[9])$.

The real pole placement map is harder to study. For a survey of early results we refer to $[2,12]$. X. Wang [16] proved that $n<m p$ is sufficient for generic surjectivity of real (or complex) pole placement map. A simplified proof of this result can be found in $[17,12]$.

From now on we only discuss the so-called critical case, that is we assume

$$
n=m p
$$

in the rest of the paper. In addition, we may assume without loss of generality that $p \leq m$, in view of the symmetry of our problem with respect to the interchange of $m$ and $p$ (see, for example, [15, Theorem 3.3]).

One corollary from Theorem A is that the real pole placement map is generically surjective if $d(m, p)$ is odd. This number is odd if and only if one of the following conditions is satisfied [2]: a) $\min \{m, p\}=1$, or b) $\min \{m, p\}=2$, and $\max \{m, p\}+1$ is an integral power of 2 .

In the opposite direction, Willems and Hesselink [18] found by explicit computation that the real pole placement map is not generically surjective for $(m, p)=(2,2)$. A closely related fact, that the problem of enumerative geometry mentioned above, may have no real solutions for the case $(m, p)=(2,2)$ even when the given 2-subspaces are real, is mentioned in [8].

In [13] Rosenthal and Sottile found with a rigorous computer-assisted proof that the real pole placement map is not generically surjective in the case $(m, p)=(4,2)$, thus disproving a conjecture of $\mathrm{Kim}$, that $(2,2)$ is the only exceptional case.

In [6] we showed that the real pole placement map is not generically surjective when $p=2$ and $m$ is even, thus extending the negative results for the cases $(2,2)$ and $(4,2)$ stated above.

In the present paper we extend this result to all cases when both $m$ and $p$ are even.

Theorem 1 If $n=m p$, and $m$ and $p$ are both even, then the real pole placement map is not generically surjective.

Our proof of Theorem 1 gives explicitly a system $S_{0} \in \mathfrak{A}$, and a polynomial $u(s)=s\left(s^{2}+1\right)^{m p / 2-1}$, such that for any $S^{\prime}$ in a neighborhood of $S_{0}$, the real pole placement map $\chi_{S^{\prime}}$ omits a neighborhood of $u$.

Our proofs in [6] depend on a hard analytic result from [5], related to the so-called B. and M. Shapiro conjecture which is stated below in Section 2. The proofs in the present paper are new, even in the case $\min \{m, p\}=2$, and they are elementary.

We conclude the Introduction with an unsolved problem.

A system $S$ is called stabilizable (by real static output feedback), if there exists a gain matrix $K \in \operatorname{Mat}_{\mathbf{R}}(m \times p)$ such that all zeros of the closed loop characteristic polynomial $\varphi_{K}$ belong to the left half-plane. From the positive results on pole placement stated above, it follows that generic systems with $m$ inputs, $p$ outputs and state of dimension $n$ are stabilizable if $n<m p$, or if $n=m p$ and $d(m, p)$ is odd. We 
ask whether generic systems with $n=m p$ and even $m$ and $p$ are stabilizable. The answer is known to be negative in the case $(m, p)=(2,2)$ [3]. For complex output feedback, with static or dynamic compensators, the problem of generic stabilizability was solved in [10].

We thank the referees of this paper for their helpful comments.

2. A class of linear systems. We begin with a well-known transformation of the closed loop characteristic polynomial (1.3). The open loop transfer function of a system of McMillan degree $n$, equal to the dimension of the state space, can be factorized as

$$
C(s I-A)^{-1} B=D(s)^{-1} N(s), \quad \operatorname{det} D(s)=\operatorname{det}(s I-A),
$$

where $D$ and $N$ are polynomial matrix-functions of sizes $p \times p$ and $p \times m$, respectively. For the possibility of such factorization for systems (1.1) of McMillan degree $n$ we refer to [4, Assertion 22.6]. Using (2.1), and the identity $\operatorname{det}(I-P Q)=\operatorname{det}(I-Q P)$, which is true for all rectangular matrices of appropriate dimensions, we write

$$
\begin{aligned}
\varphi_{K}(s) & =\operatorname{det}(s I-A-B K C)=\operatorname{det}(s I-A) \operatorname{det}\left(I-(s I-A)^{-1} B K C\right) \\
& =\operatorname{det}(s I-A) \operatorname{det}\left(I-C(s I-A)^{-1} B K\right) \\
& =\operatorname{det} D(s) \operatorname{det}\left(I-D(s)^{-1} N(s) K\right)=\operatorname{det}(D(s)-N(s) K) .
\end{aligned}
$$

This can be rewritten as

$$
\varphi_{K}(s)=\operatorname{det}\left([D(s), N(s)]\left[\begin{array}{c}
I \\
-K
\end{array}\right]\right) .
$$

Now we extend $\chi_{S}: K \mapsto \varphi_{K}$ to a map between compact manifolds. For this purpose, we allow an arbitrary $(m+p) \times p$ complex matrix $L$ of rank $p$ in (2.2) instead of

$$
\left[\begin{array}{c}
I \\
-K
\end{array}\right]
$$

and define

$$
\varphi_{L}(s)=\operatorname{det}([D(s), N(s)] L)
$$

A system $S$ represented by $[D(s), N(s)]$ is called non-degenerate if $\varphi_{L} \neq 0$ for every $(m+p) \times p$ matrix $L$ of rank $p$. Such matrices are called equivalent, $L_{1} \sim L_{2}$ if $L_{1}=L_{2} U$ where $U \in G L_{p}(\mathbf{C})$. The set of equivalence classes is the Grassmannian $G_{\mathbf{C}}(p, m+p)$ which is a compact algebraic manifold of dimension $m p$. If $L_{1} \sim L_{2}$, we have $\varphi_{L_{1}}=c \varphi_{L_{2}}$, where $c \neq 0$ is a constant. The space of all non-zero polynomials of degree at most $m p$, modulo proportionality, is identified with the projective space $\mathbf{C P}^{m p}$, coefficients of the polynomials serving as homogeneous coordinates. Monic polynomials represent the points of an open dense subset of $\mathbf{C P}^{m p}$, a so-called "big cell", which consists of polynomials of degree $m p$. This construction extends the complex pole placement map of a non-degenerate system to a regular map of compact algebraic manifolds

$$
\chi_{S}: G_{\mathbf{C}}(p, m+p) \rightarrow \mathbf{C P}^{m p},
$$

where $\chi_{S}(L)$ is the proportionality class of the polynomial $\varphi_{L}$ in (2.4), and $L$ is a matrix of rank $p$ representing a point in $G_{\mathbf{C}}(p, m+p)$. The set $\mathfrak{B}$ all non-degenerate systems is open and dense in the set $\mathfrak{A}$ of all systems, and the map

$$
X \times G_{\mathbf{C}}(p, m+p) \rightarrow \mathbf{C P}^{m p}, \quad(S, L) \mapsto \chi_{S}(L)
$$


is continuous. Notice that the subset of $G_{\mathbf{R}}(p, m+p)$ consisting of points which can be represented by matrices $L$ of the form (2.3) is open and dense. It corresponds via $\chi_{S}$ to the big cell in $\mathbf{C} \mathbf{P}^{m p}$ consisting of polynomials of degree $m p$.

We consider a system $S_{0}=\left(A_{0}, B_{0}, C_{0}\right)$ represented by the following polynomial matrix $[D(s), N(s)]$

$$
=\left[\begin{array}{ccccc}
1 & s & \ldots & s^{m+p-2} & s^{m+p-1} \\
0 & 1 & \ldots & (m+p-2) s^{m+p-3} & (m+p-1) s^{m+p-2} \\
\ldots & \ldots & \ldots & \ldots & \ldots \\
0 & 0 & \cdots & \cdots & (m+1) \ldots(m+p-1) s^{m}
\end{array}\right]
$$

The first row of $[D(s), N(s)]$ consists of monic monomials, and the $k$-th row is the $(k-1)$-st derivative of the first, for $2 \leq k \leq p$. This system $S_{0}$ has McMillan degree $m p$, and the matrices $A_{0}, B_{0}, C_{0}$ can be recovered from [ $\left.D, N\right]$, by [4, Theorem 22.18]. Let $L=\left(a_{i, j}\right)$. Introducing polynomials

$$
f_{j}(s)=a_{1, j}+a_{2, j} s+\ldots+a_{m+p-1, j} s^{m+p-2}+a_{m+p, j} s^{m+p-1},
$$

for $1 \leq j \leq p$, we can write $(2.4)$ as

$$
\varphi_{L}=W\left(f_{1}, \ldots, f_{p}\right)=\left|\begin{array}{ccc}
f_{1} & \cdots & f_{p} \\
f_{1}^{\prime} & \cdots & f_{p}^{\prime} \\
\ldots & \cdots & \ldots \\
f_{1}^{(p-1)} & \cdots & f_{p}^{(p-1)}
\end{array}\right|
$$

Thus for our system $\left(A_{0}, B_{0}, C_{0}\right)$, the pole placement map becomes the Wronski map, which sends a $p$-vector of polynomials into their Wronski determinant. We say that two $p$-vectors of polynomials are equivalent, $\left(f_{1}, \ldots, f_{p}\right) \sim\left(g_{1}, \ldots, g_{p}\right)$, if $\left(g_{1}, \ldots, g_{p}\right)=\left(f_{1}, \ldots, f_{p}\right) U$, where $U \in G L_{p}(\mathbf{C})$. Equivalent $p$-vectors have proportional Wronski determinants. Equivalence classes of $p$-vectors of linearly independent polynomials of degree at most $m+p-1$ parametrize the $\operatorname{Grassmannian} G_{\mathbf{C}}(p, m+p)$. A $p$-vector of complex polynomials will be called real if it is equivalent to a $p$-vector of real polynomials. The system represented by (2.7) is non-degenerate. This is a consequence of the well-known fact that the Wronski determinant of $p$ polynomials is zero if and only if the polynomials are linearly dependent.

To prove Theorem 1, we use the following general result (compare [13, Thm. 3.1]):

Proposition 1. If, for some $(m, n, p)$, there exists a real non-degenerate system $S_{0}=\left(A_{0}, B_{0}, C_{0}\right)$ such that the real pole placement map $\chi_{S_{0}}$ in $(2.5)$ is not surjective, then for these $(m, n, p)$ the real pole placement map is not generically surjective.

Indeed, if $\chi_{S_{0}}$ omits one point $u$, it omits a neighborhood of $u$, because the image of a compact space under a continuous map is compact. Using continuity of the map (2.6) we conclude that for all $S$ in a neighborhood of $S_{0}$ the maps $\chi_{S}$ omit a neighborhood of $u$.

In view of Proposition 1, to prove Theorem 1, it is enough to find a non-zero real polynomial of degree at most $m p$ which cannot be represented as the Wronski determinant of $p$ real polynomials of degree at most $m+p-1$. Thus Theorem 1 follows from Proposition 1 and 
Proposition 2. If $m \geq p \geq 2$ are even integers, then the polynomial $u(s)=s\left(s^{2}+\right.$ $1)^{m p / 2-1}$ is not proportional to the Wronski determinant of any $p$ real polynomials of degree at most $m+p-1$.

Proposition 2 is motivated by a conjecture of B. and M. Shapiro (see, for example [15]), which says: If the Wronskian determinant of a polynomial p-vector has only real roots, then this $p$-vector is real. In [5] we proved this conjecture for $p=2$, and used this result in [6] to derive the case $p=2$ of Theorem 1 . In the present paper, we prove a result, Proposition 3 in Section 3, which is a very special case of the B. and M. Shapiro conjecture, but still it permits to derive Proposition 2.

3. The Wronski map. A $p$-vector of linearly independent polynomials of degree at most $m+p-1$ can be represented by a $(m+p) \times p$ matrix $L$ of rank $p$, whose columns are composed of the coefficients of the polynomials as in (2.8).

The group $G L_{p}(\mathbf{C})$ acts on such matrices by multiplication from the right. This action is equivalent to the usual column operations on matrices: interchange of two columns, multiplication of a column by a non-zero constant, and adding to a column a multiple of another column. For each column $j$ of $L$, we introduce two integers $1 \leq e_{j} \leq d_{j} \leq m+p$, which are the positions of the first and last non-zero elements of this column, counted from above. Thus $\operatorname{deg} f_{j}=d_{j}-1$, and the order of a root of $f_{j}$ at zero is $e_{j}-1$. It is easy to see that by column operations, every $(m+p) \times p$ matrix $L=\left(a_{i, j}\right)$ of rank $p$ can be reduced to the following

canonical form:

(i) $d_{1}>d_{2}>\ldots>d_{p}$,

(ii) $a_{e_{j}, j}=1$, for every $j \in[1, p]$,

(iii) $a_{e_{k}, j}=0$ for $1 \leq j<k \leq p$.

The elements $a_{e_{j}, j}=1,1 \leq j \leq p$ of the canonical form will be called the pivot elements. It follows from (iii) that all numbers $e_{j}$ are distinct.

Proposition 3. Suppose that mp is even. Then every polynomial p-vector $\left(f_{1}, \ldots, f_{p}\right)$ of degree at most $m+p-1$ in canonical form, which satisfies

$$
W\left(f_{1} \ldots, f_{p}\right)=\lambda w, \quad \text { where } \quad w(s)=s^{m p / 2+1}-s^{m p / 2-1}, \quad \lambda \in \mathbf{C}^{*}
$$

has only real entries.

Corollary. All polynomial p-vectors of degree at most $m+p-1$ satisfying (3.1) are real.

This Corollary confirms a special case of the B. and M. Shapiro Conjecture, when the Wronskian determinant of a polynomial $p$-vector is $w(s)=s^{m p / 2+1}-s^{m p / 2-1}$, which is a polynomial with real roots $0, \pm 1$.

The properties of the Wronskian determinants used here are well-known and easy to prove:

Lemma. The Wronski map $\left(f_{1}, \ldots, f_{p}\right) \mapsto W\left(f_{1}, \ldots, f_{p}\right)$ is linear with respect to each $f_{j}$, and

$$
W\left(s^{n_{1}}, \ldots, s^{n_{p}}\right)=V\left(n_{1}, \ldots, n_{p}\right) s^{n_{1}+\ldots+n_{p}-p(p-1) / 2},
$$


where

$$
V\left(n_{1}, \ldots, n_{p}\right)=\prod_{k<j}\left(n_{j}-n_{k}\right)
$$

is the Vandermonde determinant.

Using this Lemma, we compute the Wronskian determinant of a polynomial $p$ vector in canonical form, and conclude that

$$
\operatorname{deg} W\left(f_{1}, \ldots, f_{p}\right)=d_{1}+\ldots+d_{p}-p(p+1) / 2,
$$

and

$$
\operatorname{ord} W\left(f_{1}, \ldots, f_{p}\right)=e_{1}+\ldots+e_{p}-p(p+1) / 2
$$

where ord denotes the multiplicity of a root at zero.

Proof of Proposition 3. According to (3.1), $\operatorname{deg} w=m p / 2+1$, and $\operatorname{ord} w=$ $m p / 2-1$. So (3.2) and (3.3) imply

$$
\begin{aligned}
& d_{1}+\ldots+d_{p}=p(p+1) / 2+m p / 2+1, \\
& e_{1}+\ldots+e_{p}=p(p+1) / 2+m p / 2-1 .
\end{aligned}
$$

Subtracting the second equation from the first, we get

$$
\sum_{j=1}^{p}\left(d_{j}-e_{j}\right)=2 .
$$

As all the summands are non-negative, there are two possibilities:

Case 1. In all columns but one, all elements, except the pivot elements, are equal to zero, and for the exceptional column $j, d_{j}-e_{j}=2$. Computing the Wronskian and comparing with (3.1), we obtain

$$
\begin{aligned}
& V\left(\ldots, e_{j}-1, \ldots\right) s^{m p / 2-1} \\
+ & V\left(\ldots, e_{j}, \ldots\right) a_{e_{j}+1, j} s^{m p / 2} \\
+ & V\left(\ldots, e_{j}+1, \ldots\right) a_{e_{j}+2, j} s^{m p / 2+1} \\
= & -\lambda s^{m p / 2-1}+\lambda s^{m p / 2+1} .
\end{aligned}
$$

Here and in what follows, the notation $V\left(\ldots, e_{j}+m, \ldots\right)$ means the Vandermonde determinant of $p$ arguments, whose $k$-th argument is $e_{k}-1$ for $k \neq j$, and the $j$-th argument is $e_{j}+m$.

Comparing the terms with $s^{m p / 2-1}$ we conclude that $\lambda$ is real. Comparing the terms with $s^{m p / 2+1}$ we conclude that $V\left(\ldots, e_{j}+1, \ldots\right) \neq 0$, and thus $a_{e_{j}+2, j}$ is real. Now we consider the middle term in the expansion of the Wronskian determinant. If $V\left(\ldots, e_{j}, \ldots\right)=0$ then $e_{k}=e_{j}+1$ for some $k$. As $d_{k}=e_{k}$, and $d_{j}=e_{j}+2$, we conclude that $d_{k}=d_{j}-1$, so $k>j$ by (i) in the definition of the canonical form. Now (iii) from the definition of the canonical form implies that $a_{e_{j}+1, j}=0$. If $V\left(\ldots, e_{j}, \ldots\right) \neq 0$, we also conclude that $a_{e_{j}+1, j}=0$. Thus all entries of $L$ are real. 
Case 2. In all columns but two, all non-pivot elements are equal to zero, and the two exceptional columns contain one extra non-zero element each. Let $j<k$ be the positions of the exceptional columns, and $a=a_{e_{j}+1, j}$ and $b=a_{e_{k}+1, k}$ the non-zero, non-pivot elements of these columns. Computing the Wronskian and comparing with (3.1), we obtain

$$
\begin{aligned}
& V\left(\ldots, e_{j}-1, \ldots\right) s^{m p / 2-1} \\
+ & \left(a V\left(\ldots, e_{j}, \ldots\right)+b V\left(\ldots, e_{k}, \ldots\right)\right) s^{m p / 2} \\
+ & a b V\left(\ldots, e_{j}, \ldots, e_{k}, \ldots\right) s^{m p / 2+1} \\
= & -\lambda s^{m p / 2-1}+\lambda s^{m p / 2+1},
\end{aligned}
$$

where $V\left(\ldots, e_{j}, \ldots, e_{k} \ldots\right)$ denotes the Vandermonde determinant of $p$ arguments, whose $j$-th argument is $e_{j}, k$-th argument is $e_{k}$ and for all other indices $l \notin\{j, k\}$, the $l$-th argument is $e_{l}-1$.

Our first conclusions are

$$
V\left(\ldots, e_{j}-1, \ldots\right)=-\lambda
$$

and

$$
V\left(\ldots, e_{j}, \ldots, e_{k}, \ldots\right) \neq 0
$$

It follows from $(3.5)$ that $\lambda$ is real. If exactly one of the numbers $V\left(\ldots, e_{j}, \ldots\right)$ and $V\left(\ldots, e_{k}, \ldots\right)$ is zero, then (3.4) implies that at least one of the numbers $a$ or $b$ is zero. Then the third term in the expansion of the Wronskian is zero, which contradicts (3.4). If both $V\left(\ldots, e_{j}, \ldots\right)$ and $V\left(\ldots, e_{k}, \ldots\right)$ are zero, then $V\left(\ldots, e_{j}, \ldots, e_{k}, \ldots\right)=$ 0 , and this contradicts (3.6). So both $V\left(\ldots, e_{j}, \ldots\right)$ and $V\left(\ldots, e_{k}, \ldots\right)$ are nonzero. This means that there are no pivot elements in the rows $e_{j}+1$ and $e_{k}+1$. Using (3.6) we conclude that $V\left(\ldots, e_{j}-1, \ldots\right), V\left(\ldots, e_{j}, \ldots\right), V\left(\ldots, e_{k}, \ldots\right)$ and $V\left(\ldots, e_{j}, \ldots, e_{k}, \ldots\right)$ have the same sign, and by $(3.5)$, all these numbers have the sign of $-\lambda$. As $V\left(\ldots, e_{j}, \ldots\right)$ and $V\left(\ldots, e_{k}, \ldots\right)$ are of the same sign, (3.4) implies that $a=-c b$, where $c>0$, and from the equations

$$
V\left(\ldots, e_{j}, \ldots, e_{k}, \ldots\right) a b=\lambda
$$

and (3.5) we conclude that $a$ and $b$ are real.

The group Aut $\left(\mathbf{C P}^{1}\right)$ of fractional-linear transformations acts on the space $\mathbf{C P}^{k}$ of proportionality classes of non-zero polynomials of degree at most $k$ by the following rule. Let

$$
\ell(s)=\frac{a s+b}{c s+d}, \quad a d-b c \neq 0
$$

represent a fractional-linear transformation. For a polynomial $r(s)$, we put

$$
\operatorname{lr}(s)=(-c s+a)^{k} r \circ \ell^{-1}(s) .
$$

That this is indeed a group action, can be verified as follows. The space of proportionality classes of non-zero polynomials of degree at most $k$ can be canonically identified with the symmetric power $\operatorname{Sym}^{k}\left(\mathbf{C P}^{1}\right)$, which is the set of unordered $k$-tuples of 
points in $\mathbf{C P}^{1}$. To each polynomial $r$ one puts into correspondence its roots, counted with multiplicity, and the point $\infty$ with multiplicity $k-\operatorname{deg} r$. Then the action of $\ell \in \operatorname{Aut}\left(\mathbf{C P}^{1}\right)$ on such $k$-tuple is simply

$$
\left(s_{1}, \ldots, s_{k}\right) \mapsto\left(\ell\left(s_{1}\right), \ldots, \ell\left(s_{k}\right)\right) .
$$

It is easy to verify that this action of $\operatorname{Aut}\left(\mathbf{C P}^{1}\right)$ extends to the space $G_{\mathbf{C}}(p, m+p)$ of equivalence classes of polynomial $p$-vectors of degree at most $m+p-1$. Furthermore, this extended action is respected by the Wronski map:

$$
W\left(\ell g_{1}, \ldots, \ell g_{p}\right)=\ell W\left(g_{1}, \ldots, g_{p}\right) .
$$

Of course, in the left hand side of this equality, the group $\operatorname{Aut}\left(\mathbf{C P}^{1}\right)$ acts on $\operatorname{Sym}^{m+p-1}\left(\mathbf{C P}^{1}\right)$, while in the right hand side it acts on $\operatorname{Sym}^{m p}\left(\mathbf{C P}^{1}\right)$. Equation (3.7) permits to simplify the polynomial equation

$$
W\left(g_{1}, \ldots, g_{p}\right)=v, \quad v(s) \sim s\left(s^{2}-1\right)^{m p / 2-1}
$$

which will be used to prove Proposition 2 .

Consider the fractional-linear transformation

$$
\ell(s)=\ell^{-1}(s)=\frac{1-s}{1+s} .
$$

We have $\ell:(0,1, \infty,-1) \mapsto(1,0,-1, \infty)$, and $\ell(\overline{\mathbf{R}})=\overline{\mathbf{R}}$.

Using (3.8) and (3.9) we obtain

$$
\ell v(s)=(s+1)^{m p} v \circ \ell^{-1}(s) \sim s^{m p / 2+1}-s^{m p / 2-1}=w(s),
$$

where " $\sim$ " means "proportional". Thus, with $f_{j}=\ell g_{j}$, the equation (3.8) is equivalent to the equation

$$
W\left(f_{1}, \ldots, f_{p}\right)=w, \quad w(s) \sim s^{m p / 2+1}-s^{m p / 2-1},
$$

which we solved in Proposition 3. The conclusion is that

$$
\text { all solutions of (3.8) in canonical form have real coefficients. }
$$

Proof of Proposition 2. Suppose that $\left(f_{1}, \ldots, f_{p}\right)$ is a real polynomial $p$-vector in canonical form satisfying

$$
W\left(f_{1}, \ldots, f_{p}\right)=u, \quad u(s)=\lambda s\left(s^{2}+1\right)^{m p / 2-1}, \quad \lambda \neq 0 .
$$

Then (3.3) implies

$$
e_{1}+\ldots+e_{p}=1+p(p+1) / 2,
$$

As $\left(e_{j}\right)_{j=1}^{p}$ are distinct positive integers, the only possibility is

$$
\left\{e_{1}, \ldots, e_{p}\right\}=\{1,2 \ldots, p-1, p+1\} .
$$

Similarly, (3.2) implies

$$
d_{1}+\ldots+d_{p}=m p+p(p+1) / 2-1 .
$$


As $\left(d_{j}\right)_{j=1}^{p}$ are distinct integers in the interval $[1, m+p]$, the only possibility is that

$$
\left\{d_{1}, \ldots, d_{p}\right\}=\{m, m+2, m+3, \ldots, m+p\} .
$$

Notice that the sequence (3.13) contains $p / 2+1$ odd numbers and $p / 2-1$ even numbers. On the other hand, the sequence (3.14) contains $p / 2-1$ odd numbers and $p / 2+1$ even numbers. This implies that at least for one $j$

$$
d_{j}-e_{j} \text { is odd. }
$$

This means that the polynomial $f_{j}$ contains both even and odd powers of $s$ with non-zero coefficients. So the polynomial $g_{j}(s)=f_{j}(i s), i=\sqrt{-1}$, is not proportional to any polynomial with real coefficients. On the other hand, the polynomial $p$-tuple $\left(g_{1}, \ldots, g_{p}\right)$, where $g_{j}(s)=\epsilon_{j} f_{j}(i s)$ with appropriate $\epsilon_{j} \in\{ \pm 1, \pm i\}$ is a solution of (3.8) in canonical form, and we know from (3.11) that all such solutions have real coefficients. This contradiction completes the proof of Proposition 2.

\section{REFERENCES}

[1] R. Brockett and C. Byrnes, Multivariable Nyquist criteria, root loci and pole placement: a geometric viewpoint, IEEE Trans. Aut. Contr. AC-26 (1981) 271-284.

[2] C. BYRnES, Pole assignment by output feedback, in Three decades of mathematical system theory, H. Nijmeijer and J.M. Schumacher, eds. Lect. Notes Contr. and Inf. Sci. 135, 31-78. Springer Verlag, 1989.

[3] C. Byrnes, B. Anderson, Output feedback and generic stabilizability, SIAM J. Control Optim., 22 (1984) 362-280.

[4] D. Delchamps, State space and input-output linear systems, Springer, NY, 1988.

[5] A. ERemenko And A. Gabrielov, Rational functions with real critical points and the B. and M. Shapiro conjecture in real enumerative geometry, to appear in Ann. Math.

[6] A. Eremenko And A. Gabrielov, Counterexamples to pole placement by static output feedback, to appear in Linear Algebra and Appl.

[7] R. Hermann and C. Martin, Applications of algebraic geometry to systems control theory, part I, IEEE Trans. Automat. Control, AC-22 (1977) 19-25.

[8] S. Kleiman, Problem 15: Rigorous foundation of Schubert's enumerative calculus, in: F. Browder, ed., Mathematical development arising from Hilbert problems, AMS, Providence RI, 1976.

[9] S. Kleiman and D. Laksov, Schubert calculus, Amer. Math. Monthly 79 (1972) 1061-1082.

[10] M. Ravi, J. Rosenthal, X. Wang, On generic stabilizability and pole assignability, Systems and Control Letters, 23 (1994) 79-84.

[11] M. Ravi, J. Rosenthal, X. Wang, Dynamic pole assignment and Schubert calculus, SIAM J. Control Optim., 34 (1996) 813-832.

[12] J. Rosenthal, J. Schumacher and J. Willems, Generic eigenvalue assignment by memoryless output feedback, Syst. Control Lett. 26 (1995) 253-260.

[13] J. Rosenthal And F. Sottile, Some remarks on real and complex output feedback, Systems Control Lett. 33 (1998) 73-80.

[14] J. Rosenthal, X. WAng, Output feedback pole placement with dynamic compensators, IEEE Trans. Aut. Contr., 41 (1996) 830-843.

[15] F. SotтiLe, Real Schubert calculus: polynomial systems and a conjecture of Shapiro and Shapiro, Exper. Math., 9 (2000) 161-182.

[16] X. WAng, Pole placement by static output feedback, J. Math. Systems, Estimation and Control, 2 (1992) 205-218,

[17] X. WANG, Grassmannian, central projection and output feedback pole assignment of linear systems, IEEE Trans. Automat. Control, 41 (1996) 786-794.

[18] J. Willems and W. Hesselink, Generic properties of the pole placement problem, Proc. 7-th IFAC Congress, (1978) 1725-1728. 